Special Issue on KEER 2012

ORIGINAL ARTICLE

\title{
The Influence of Pictorial Reality on Evaluation of Appropriate Force Feedback
}

\author{
Manako TAI*, Kazuaki KOJIMA** and Tatsunori MATSUI** \\ * Graduate School of Human Sciences, Waseda University 2-579-15 Mikajima, Tokorozawa, Saitama 359-1192, Japan \\ ** Faculty of Human Sciences, Waseda University 2-579-15 Mikajima, Tokorozawa, Saitama 359-1192, Japan
}

\begin{abstract}
In addition to the visual and auditory modalities, future media communication is expected to provide information through the tactile modality. To achieve effective tactile media in daily life settings, it is important to study its representation from the aspects of human cognition and emotion. This study experimentally investigated the effects of visual representation on evaluations of tactile information. We conducted an experiment in which subjects were asked to evaluate the appropriateness of tactile information of foods presented on a monitor after touching them using PHANTOM Omni, a haptical device. Textures (photos or paintings) of the foods and the intensity of force feedback were controlled. The results did not clearly confirm the influences of the visual representation on evaluation of the force feedback, even though it indicated the possibility that force feedback can be more clearly distinguished when the stimuli are represented by paintings rather than by photos.
\end{abstract}

Keywords: Visual and haptic media, the reality of visual information, tactile information, evaluation of impression

\section{INTRODUCTION}

\subsection{Research Background}

One major form of media communication in modern society is visuo-audio media, in which information is conveyed thorough visual and auditory modalities. Due to the Internet and globalization, communication through electronic media has increasingly been used in a variety of contexts, such as electronic commerce and teleconferences. As telecommunication has widely increased, it is expected that future electronic media will need to increase modalities to provide further information that cannot be transferred through visual and auditory modalities. Tactile information is provided through one of those modalities, which is defined in the field of physiology as somatic sensations, which include cutaneous and deep sensations. In fact, new media capable of presenting tactile information has been studied, such as a touch panel that can alter tactile sensation of its surface, such as rough or uneven textures [8] and a virtual museum where users can touch artwork [1]. Therefore, future electronic media must be endowed with tactile modality. Accordingly, it has become an important task to study media that include tactile information.

Several studies have already addressed tactile media. One major task of these studies is to develop virtual reality (VR) systems to support skill acquisition in particular domains, such as a simulator for medical operations [6] and distance education for calligraphy [4]. It is critical for such VR systems to precisely reproduce the real world because they have to offer experiences that are identical to those in the real world. To achieve this, the systems require reality; they must represent visual information of objects as if they are the real objects. Here, the reality of information represented in a medium denotes the degree of how precisely the information about real objects is reproduced. Thus, photos as representation of visual information have high reality, while paintings have low reality. High reality information representation does not always affect people positively in daily life settings; it can negatively affect human cognition and emotion. One example of the negative effects produced by high reality in visual representation is a phenomenon whereby people feel unfamiliar to humanoid robots whose external representation is considerably similar to humans, which was documented as the theory of uncanny valley by Mori [5]. It hypothesizes that people's perceptions of robots are more favorable as the robots appear more humanlike; however, a robot with an exceptionally human-like appearance gives people a sense of fear and revulsion causing its familiarity drastically decrease to the bottom of the valley. Another example is found in educational research, which has studied the relationship between the presentation of information in educational materials and their effects on learning. Yanagisawa and Akahori [9] 
gave their subjects the task of familiarizing themselves with the structure of a university campus, whose layout was represented by photos depicting images of the actual campus or paintings that did not include such images. They proved that task performance was higher when subjects viewed the paintings than when they viewed the photos. They argued that the cognitive load becomes heavier when the reality of the visual information is higher and that task performance can suffer from the load.

In human information processing, visual information precedes other forms of information [2,7]. This phenomenon is known as visual domination and can occur when people use visual and tactile media [3]. Because too much visual information may implicitly impose a heavy cognitive load on its receiver, his or her processing of tactile information can be disrupted. To prevent human cognition from suffering from a heavy cognitive load, therefore, it is important for the visual and tactile media to control the amount of visual information given at one time.

\subsection{Purpose of this Study}

Based on the background information thus presented, the current study addressed the issue of representation of visual and tactile information. Because our focus was on the psychological aspect of communication, we experimentally investigated the effects of the reality of visual information on evaluations of tactile sensation in visuo-tactile media. We then verified that high reality of visual information disrupted tactile sensation.

\section{METHOD}

\subsection{Materials}

We composed visuo-tactile media that presented stimuli created by combining images displayed on a personal computer (PC) monitor and force feedback generated by a haptical device of PHANTOM Omni, which can provide force feedback, enabling a user to touch a virtual object. The force feedback from the virtual object is sent to the user through a stylus that serves as a pointing device. When the stylus approached the virtual object's surface, the force was produced on the surface on the object as if a virtual spring on the surface had pressed the tip of the stylus. The intensity of the force feedback is computed with Hooke's law, which is based on the spring rate of a virtual spring and its compression distance.

In the experiment, stimuli presented in the media were five foods: an apple, an orange, a Japanese pear, a chestnut, and a caramel, which are shown in Figure 1. Visual information of the foods was controlled from the aspect of the reality of their surface textures, and tactile information was controlled by the intensity of force feedback from PHANTOM Omni. Each food was represented as a 3-D graphic object whose texture map was made from either a photo or painting. We refer to photo textures as $\mathrm{Tp}$ and painting textures as Ti. The painting textures were produced by simplifying gradations and patterns of photos to the extent that each food could be discriminated. Tactile information of the foods was force feedback, whose intensity was one of four levels of spring rates: $0.25,0.5,0.75$, or 1.0 . We refer to the force feedback of those spring rates as F1, F2, F3, and F4. Ranges where the force feedback was exerted were set up for each stimulus according to the shape and size of its food. The ranges were spheres with 0.5 -inch radiuses for the apple, orange, and pear, a cone a 1.4-inch height and a 1-inch radius for the chestnut, and a cube with 0.13 -inch sides for the caramel.

\subsection{Procedures}

Twenty undergraduate students ( 7 males and 13 females whose average age was 22.7) participated in the experiment. In each trial of an experimental task, the subjects were shown a pair of stimuli in the media and asked to choose the more appropriate tactile sensation of the two for the food after touching them using the stylus of PHANTOM Omni. Each pair comprised two stimuli that had the same food and the same texture, but their force feedback was different. The subjects engaged in six trials to compare all combinations of force feedback of the four spring rates in each texture of each food. Thus, the entire number of trials was sixty: ten sets (the five foods and the two textures) of six trials. Figure 2 shows the experimental setting. Orders of sets of trials to evaluate stimuli with the two textures were counterbalanced. Half of the subjects engaged in trials with Ti after completing trials with $\mathrm{Tp}$, and the others in $\mathrm{Tp}$ after completing trials with Ti.

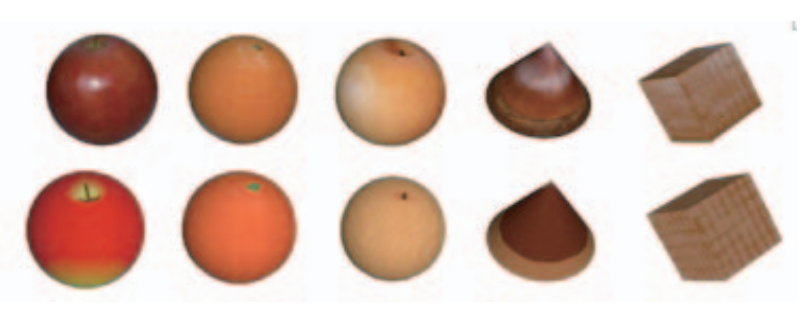

Figure 1: Foods presented as stimuli (upper row: photos, lower row: paintings) 


\section{Visual Information}

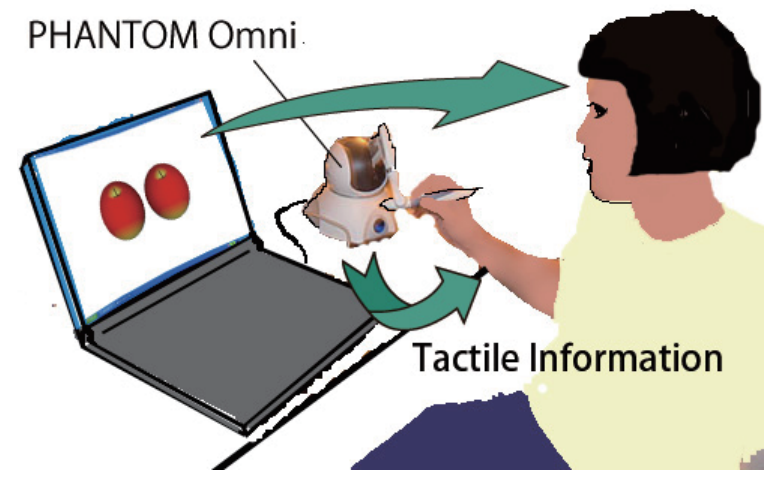

Figure 2: Experimental setting

\subsection{Analysis}

To rank the appropriateness of the force feedback of the four spring rates for each texture of each food, a distance scale was estimated from each set of six trials based on Thurstone's paired comparison. Each distance scale was composed according to the proportion of subjects who chose one of a pair in each trial. Appropriateness of the force feedback for each visual representation was studied based on the distance scales.

Furthermore, to compare evaluations among subjects, we computed ranking scores for each force feedback with the following procedures. In each set containing six trials by a subject, 1 point was added to one of a pair if it had been chosen, and -1 point was added if not chosen in every trial. We then newly scored 4 to the stimulus that had gained the highest points, 3 to the second, 2 to the third and 1 to the stimulus with the least points. Ranking scores were revised so that their total was always 10 , if needed. For example, points for the four stimuli were $(1,1,1,-3)$; their ranking scores were then revised to $(3,3,3,1)$. In the same manner, scores for points $(1,1,-1,-1)$ were revised to $(3.5,3.5,1.5,1.5)$, and those of $(3,1,1,1)$ were revised to $(4,2,2,2)$. Evaluations by each subject were studied by comparing ranking scores of the two textures.

\subsection{Hypotheses}

In our experimental task, subjects must have been able to evaluate tactile sensation based on their experiences about the foods in the real world. Therefore, we drew the following hypothesis:

i) Force feedback of spring rates gaining the highest values in the distance scales are different among the five foods.

We drew another hypothesis because high reality in visual representation can disrupt human cognition, as described in the previous section.

ii) Differences of values in distant scales among the force feedback of the four spring rates are larger in stimuli of Ti than in stimuli of Tp.

\section{RESULTS}

\subsection{Distant Scales for Each Food}

Figures 3, 4, 5, 6, and 7 show distance scales for the apple, orange, pear, chestnut, and caramel. In each scale, the magnitude of the evaluations of force feedback is relatively represented. The right side of the scales denotes that the evaluations were high; in other words, force feedback on the right side was more appropriate for tactile sensation of the foods. Line-drawing triangles point values of $\mathrm{Ti}$, and filled triangles point values of $\mathrm{Tp}$.

We conducted a two-way 2- (textures $\mathrm{Tp}$ and $\mathrm{Ti}$ ) by-4 (force feedback F1, F2, F3, and F4) analysis of variance (ANOVA) for each food. No significant results on the effects of the textures were confirmed in any of the foods

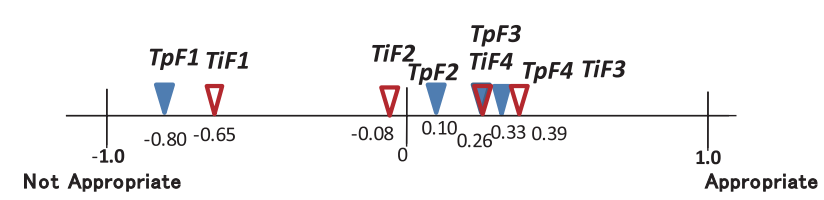

Figure 3: Distance scale of stimuli of the two textures (apple)

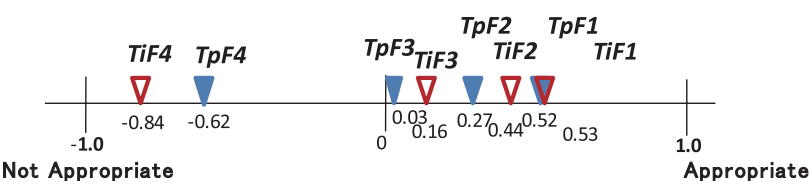

Figure 4: Distance scale of stimuli of the two textures (orange)

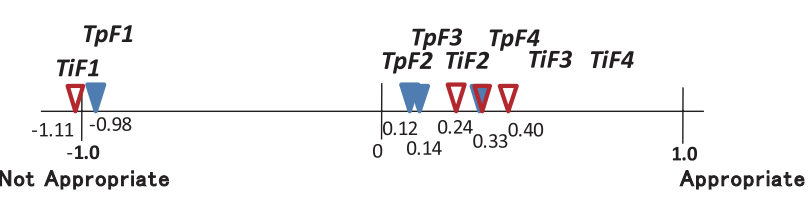

Figure 5: Distance scale of stimuli of the two textures (Japanese pear)

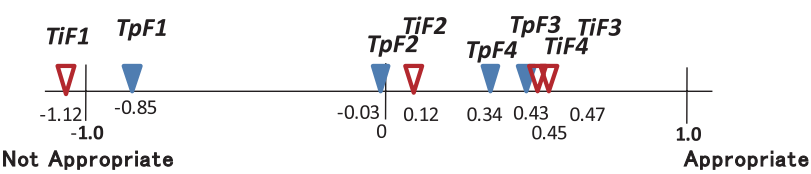

Figure 6: Distance scale of stimuli of the two textures (chestnut)

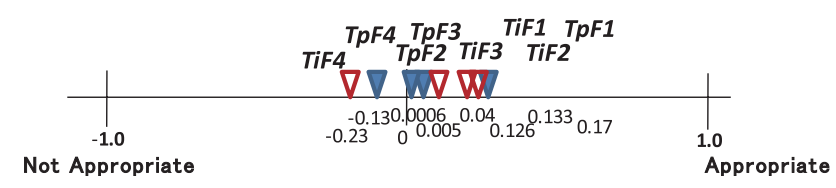

Figure 7: Distance scale of stimuli of the two textures (caramel) 
$(\mathrm{F}(1,7)=0.005$, n.s. in the apple; $\mathrm{F}(1,7)=0.04$, n.s. in the orange; $\mathrm{F}(1,7)=0.84$, n.s. in the pear; $\mathrm{F}(1,7)=0.006$, n.s. in the chestnut; and $\mathrm{F}(1,7)=0.02$, n.s. in the caramel). A main effect of force feedback was marginally significant in the caramel $(\mathrm{F}(3,7)=7.88, \mathrm{p}<.10)$, while the main effects of force feedback were significant in the other four foods $(\mathrm{F}(3,7)=35.84, \mathrm{p}<.01$ in the apple; $\mathrm{F}(3,7)=39.65, \mathrm{p}<.01$ in the orange; $\mathrm{F}(3,7)=89.07$, $\mathrm{p}=.001$ in the pear; and $\mathrm{F}(3,7)=35.07, \mathrm{p}<.01)$. These results confirm that the force feedback gained different evaluations for each. We then conducted multiple comparisons (using Tukey's honestly significant difference (HSD) test) to examine differences among force feedback, with the results indicating that there were significant differences between the force feedback gaining the lowest score and the other force feedback in each of seven sets, excluding the Ti apple. Apple photo: force feedback with a spring rate of 0.25 and 0.5 ("TpF1" and "TpF2" in Figure 3), $\mathrm{p}=0.007$; apple painting: force feedback with a spring rate of 0.25 and 0.75 ("TiF1" and "TiF3" in Figure 3), $p=0.001$; orange photo and painting: force feedback with a spring rate of 1.0 and 0.75 ("TpF4" and "TpF3", "TiF4" and "TiF3" in Figure 4), p = 0.018, p = 0; Japanese pear photo and painting: force feedback with a spring rate of 0.25 and 0.5 ("TpF1" and "TpF2", "TiF1" and "TiF2" in Figure 5), both had p-values of 0; and chestnut photo and painting: force feedback with a spring rate of 0.25 and 0.5 ("TpF1" and "TpF2", "TiF1" and "TiF2" in Figure 6), $p=0.030, p=0)$. There was a marginal significant difference between the force feedback with a spring rate of 0.25 and 1.0 ("TpF1" and "TpF4", "TiF1" and "TiF4" in Figure 7), p <.10 in both textures in the caramel. These results revealed that the most inappropriate force feedback was consistent among many subjects. However, evaluations of the other force feedback varied.

The force feedback that gained the highest values in the distance scales was different among the foods. As the figures demonstrate, stimuli with F4 gained the highest values in the distance scales in the apple (Figure 3) and pear (Figure 5), and values decreased as the spring rates of force feedback decreased. On the contrary, the orange, F1, gained the highest values, and values decreased as spring rates increased (Figure 4). F3 was the highest and F4 the second highest (Figure 6) in the chestnut. Therefore, those facts supported hypothesis 1 . In the caramel, however, differences among stimuli were small, indicating that none of the force feedback was particularly appropriate. Perhaps the subjects found difficulty in touching caramel stimuli because of their small range of force feedback compared to the other foods' stimulus and were not able to evaluate them properly.

\subsection{Differences in Evaluations between the Textures 3.2.1 Values in the Distance Scales}

As described above, the results of the ANOVA indicated no significant differences of values between the textures in the distance scales. Although there were no significant differences, force feedback that gained the lowest values was more distant from origins of the distance scales in $\mathrm{Ti}$ than in Tp. We compared this among the five foods at each level of force feedback. Figure 8 shows distance scales for each force feedback. In each part of the figure, the upper bar indicates the distance scale of a photo of food, and the lower bar indicates the distance scale of a painting of food. It demonstrates that ranges of values were wider in paintings than in photos (1.506 in a photo and 1.715 in a painting in $\mathrm{F} 1 ; 0.305$ in a photo and 0.523 in a painting in $\mathrm{F} 2 ; 0.424$ in a photo and 0.429 in a painting in F3; and 0.961 in a photo and 1.292 in a painting in $\mathrm{F} 4$ ).

\subsubsection{Ranking Scores}

We examined the differences in the rankings of force feedback between photos and paintings. The rankings evaluated by each subject were determined by the ranking scores. Based on the changes in the rankings between the textures, we divided the subjects into the following groups:

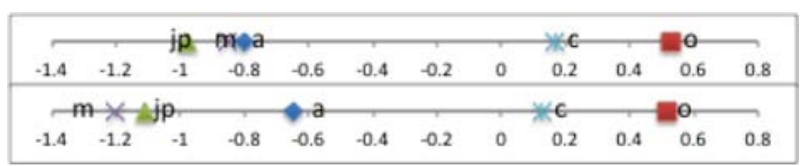

(a) Force Feedback with a spring rate of 0.25

\begin{tabular}{|c|c|c|c|c|c|c|c|}
\hline \multirow[b]{2}{*}{-0.2} & \multicolumn{4}{|c|}{$m * * c \quad a-j p$} & \multicolumn{2}{|c|}{-0} & \multirow[b]{2}{*}{0.5} \\
\hline & -0.1 & 0 & 0.1 & 0.2 & 0.3 & 0.4 & \\
\hline - & t & & $m$ & , & & T & 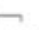 \\
\hline-0.2 & -0.1 & 0 & 0.1 & 0.2 & 0.3 & 0.4 & 0.5 \\
\hline
\end{tabular}

(b) Force Feedback with a spring rate of 0.5

\begin{tabular}{|c|c|c|c|c|c|c|c|c|c|c|}
\hline \multicolumn{3}{|c|}{$\mathrm{C}=\mathrm{O}$} & \multirow{2}{*}{$\begin{array}{l}j p \\
0.15\end{array}$} & \multicolumn{3}{|c|}{$14-a$} & & \multicolumn{3}{|c|}{$* m$} \\
\hline 0 & 0.05 & 0.1 & & 0.2 & 0.25 & 0.3 & 0.35 & 0.4 & 0.45 & 0.5 \\
\hline & $* c$ & 7 & 7 & 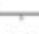 & $T$ & $T$ & $j p$ & ta & & m \\
\hline 0 & 0.05 & 0.1 & 0.15 & 0.2 & 0.25 & 0.3 & 0.35 & 0.4 & 0.45 & 0.5 \\
\hline
\end{tabular}

(c) Force Feedback with a spring rate of 0.75

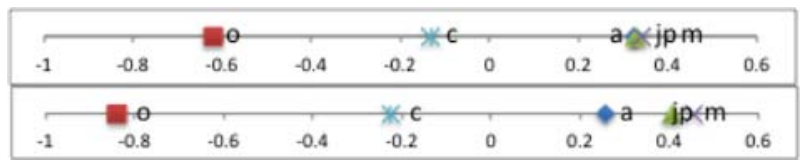

(d) Force Feedback with a spring rate of 1.0

Figure 8: Distance scales distribution in each force feedback (upper row: photo, lower row: painting)

a: apple, o: orange, jp: Japanese pear, m: chestnut, c: caramel 
All were held (A): Rankings between the textures did not change at all.

The Highest and Lowest were held (HL): Rankings of force feedback gaining the highest and lowest values did not change.

The Lowest were held (L): Rankings of the lowest values did not change, but rankings of the highest values changed.

The Highest were held $(\mathrm{H})$ : Rankings of the highest values did not change, but rankings of the lowest values changed.

Reversed (R): Rankings were entirely or gently reversed. Others $(\mathrm{O})$ : Other rankings

We assumed that the evaluations of force feedback were not influenced by the textures in Group A; Group HL was not influenced by the textures when evaluating the most appropriate and inappropriate force feedback; Group L was not influenced only when evaluating the most inappropriate force feedback; Group $\mathrm{H}$ was not influenced only when evaluating the most appropriate force feedback; and Group R was strongly influenced by the textures.

In the grouping, we assumed that only the highest force feedback was evaluated by a subject when the ranking scores were $(2,2,2,4)$, and only the lowest force feedback was evaluated when the scores were $(1,3,3,3)$. The higher two scores and lower two scores were supposed to be indicated when the ranking scores were $(1.5,1.5,3.5$, 3.5). Thus, Group R consists of subjects who switched the highest and lowest scores between the textures; those who switched the higher two and lower two scores; subjects who switched the higher two and the lowest one; and subjects who switched the lower two and the highest one. Figure 9 indicates the numbers of subjects in each group.

Subjects in Groups A, HL, and L at last held rankings of force feedback gaining the lowest values between the textures. The numbers of such subjects ranged from elev-

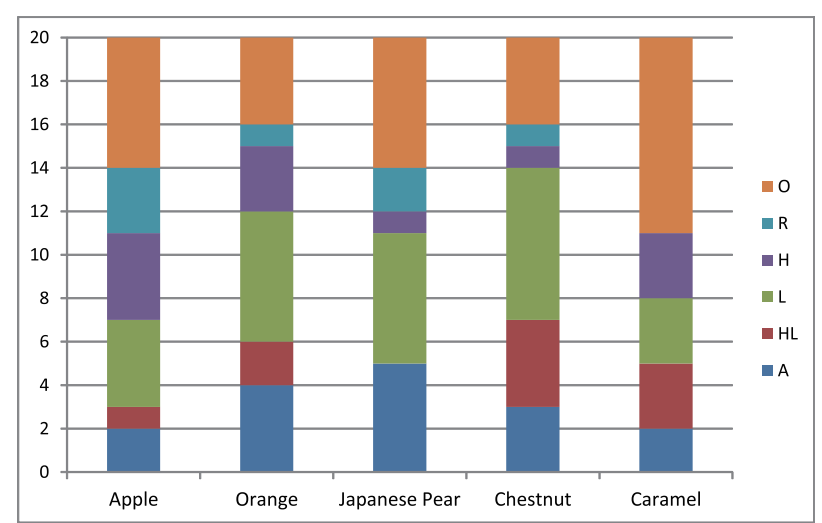

Figure 9: The numbers of subjects in each group en to fourteen in the orange, pear, and chestnut categories. The number of subjects who did not change rankings based on the textures ranged only from two to five. Thus, evaluations of force feedback basically changed, other than the one that gained the lowest values.

\section{DISCUSSION}

We had predicted that force feedback of the four spring rates was distinguished from each other more clearly when the stimuli were represented by $\mathrm{Ti}$ rather than by Tp. As indicated in the previous section, however, no significant difference between stimuli with $\mathrm{Tp}$ and $\mathrm{Ti}$ in any of the foods was found. The experimental results only demonstrated significant differences among the force feedback.

Why did the reality of the textures have no significant influence on the evaluations of tactile sensation? Perhaps this is because the subjects' prior knowledge of the foods varied. The subjects must have had a thorough knowledge of the foods resulting in their ideas about the foods' tactile sensation not being strongly affected by the visual information presented. In fact, the four force feedback rankings did not vary at all in regard to the textures in evaluations by some subjects. Furthermore, analysis of ranking scores indicated that although the most inappropriate force feedback was consistently chosen by the subjects, the other force feedback was not clearly distinguished, but varied. Force feedback F1 and F4 were obviously distinguished from the others, even though F2 and F3 were not. Therefore, it might have been difficult for the subjects. Consequently, the evaluations may not have been sufficiently influenced by the different visual representations.

Although the evaluations of the force feedback did not significantly differ between the textures, the following consistent features were observed among the subjects in the experimental results.

(1) Values of the stimuli of the lowest rankings on the distance scales were smaller in Ti than in Tp.

(2) Values on the distance scales were more divergent in $\mathrm{Ti}$ than in Tp. Accordingly, the ranges of the values were also wider in Ti than in Tp.

(3) Regardless of the textures, the stimuli gaining the lowest values in ranking scores were consistent among more than half of the subjects in the orange, pear and chestnut categories. However, orders other than the lowest ones varied in most cases. Thus, evaluations of force feedback basically changed between the textures. 
Facts (1) and (2) are consistent with hypothesis ii, indicating the possibility that force feedback can be more clearly distinguished when the stimuli are represented by the paintings rather than by the photos. Therefore, we believe that the effect of the reality of visual representation on evaluations of tactile sensation will be proven through further experimental studies. For this to occur, we have to improve the experimental method.

\section{CONCLUSIONS}

This study addressed the effects of the reality of visual representation on the evaluation of tactile sensation in visuo-tactile media. We conducted an experiment to evaluate the appropriateness of force feedback for images of familiar foods whose textures were represented in photos or paintings and confirmed the following three points:

(1) The most inappropriate force feedback was distinctly and consistently evaluated, while evaluations of the others varied.

(2) The effects of the textures were not clarified. This was considered to be due to the subjects' prior knowledge and the difficulty in distinguishing force feedback.

(3) Although differences were not significant, the evaluations of force feedback were more divergent in Ti than in Tp.

One important future work is to further study the effects of the visual representation on tactile sensation. To do this, we must improve the experimental method. For example, expanding the variety of visual and tactile representation of stimuli is necessary to overcome the difficulty in distinguishing the stimuli. In addition, further scales for evaluating the stimuli must be introduced.

\section{ACKNOWLEDGEMENTS}

This work was partially supported by a Grant-in-Aid for Challenging Exploratory Research (21650047).

\section{REFERENCES}

1. T. Asano, Y. Ishibashi, S. Minezawa; Adaptive Control on Display of Touchable Exhibits and Streaming Start of Explanatory Information in a Distributed Haptic Museum, Transactions of the Virtual Reality Society of Japan; 11(1), pp.27-37 (2006).

2. F.B. Colavita; Human Sensory Dominance, Perception \& Psychophysics; 16(2), pp.409-412 (1974).

3. A. Iesaki, A. Somada, A. Kimura, F. Shibata, H. Tamura; Psychophysical Influence on Tactual Impression by Mixed-Reality Visual Stimulation,
Transactions of the Virtual Reality Society of Japan; 13(2), pp.129-139 (2008).

4. N. Inami, H. Tominaga, Y. Matsubara, T. Yamasaki; Pencil Touch Learning System for Japanese Handwriting Based on the Haptic Device, IEICE; J87-D-1(12), pp.1128-1135 (2004).

5. M. Mori; The Uncanny Valley, Energy; 7(4), pp.33-35 (1970).

6. M. Nakao, H. Oyama, M. Komori, T. Matsuda, T. Takahashi; A Study on Visual and Haptic Display of a Beating Heart for Cardiac Surgery Simulation, Transactions of the Virtual Reality Society of Japan; 7(3), pp.413-420 (2002).

7. M.I. Posner, M.J. Nissen, R.M. Klein; Visual Dominance: An Information-processing account of its origins and significance, Psychological Review; 83(2), pp.157-171 (1976).

8. Senseg E-Sense. http://senseg.com/

9. M. Yanagisawa, K. Akahori; The Influence of Photographic Reality in Virtual Environment on Spatial Representation, Japan journal of educational technology; 22(4), pp.239-249 (1999).

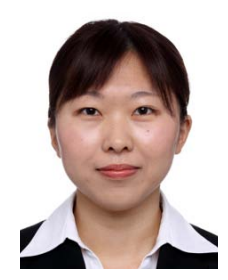

\section{Manako TAI}

Manako Tai is a graduate student in master course at Waseda University. She received B.A (2011) in Human Sciences from Waseda University. Her research interests are Human-computer Interaction and Kansei Information Science.

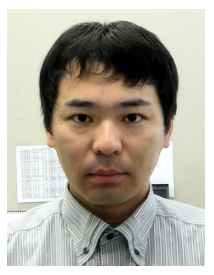

Kazuaki KOJIMA

Kazuaki Kojima is an Assistant Professor in Faculty of Human Sciences, Waseda University, Japan. He received Ph. D. in Human Science from Nagoya University in 2007. He is engaged in research of Artificial Intelligence, Cognitive Science and Educational Engineering. His research interests are in human and computational creativity. From 2007 to 2009, he was a Research Associate in Faculty of Human Sciences, Waseda University. In 2012, he won the best paper award in Japanese Society in Information and Systems in Education.

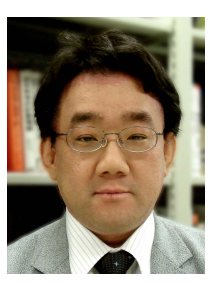

Tatsunori MATSUI

Tatsunori Matsui is a Professor of Knowledge Science and Artificial Intelligence Lab. at Faculty of Human Sciences, Waseda University, Tokyo Japan since 2004, after contributed for 7 years as an Associate Professor to Graduate School of Information Systems, the University of Electro-Communications, Tokyo Japan. Currently, his research interests are Kansei Information Science, Artificial Intelligence in Education, Mathematical Analysis for Educational Data, Statistical Science and so on. He is a member of IEEE, ACM, IEICE, IPSJ, JSAI, JSiSE and so on. 